\title{
Endoscopic Management of Severe Supraglottic and Posterior Glottic Stenosis Due to Chemotherapy and Radiation
}

\author{
Shaina M. Rubino', Michael J. Pitman² \\ ${ }^{1}$ New York Medical College, Valhalla, USA \\ ${ }^{2}$ Department of Otolaryngology, New York Eye \& Ear Infirmary, The Voice \& Swallowing Institute, New York, \\ USA \\ Email: mpitman@nyee.edu
}

Received 12 February 2014; revised 10 March 2014; accepted 8 April 2014

Copyright (C) 2014 by authors and Scientific Research Publishing Inc.

This work is licensed under the Creative Commons Attribution International License (CC BY). http://creativecommons.org/licenses/by/4.0/

(c) (i) Open Access

\begin{abstract}
Combined posterior and supraglottic stenosis (CS) often presents as debilitating dysphonia or dyspnea, secondary to blunt trauma or traumatic intubation. However, CS has proven to be a late complication of chemoradiation therapy. Traditional treatment of combined posterior and supraglottic stenosis (CS) secondary to chemoradiation has been frequently complicated by poor tissue healing. This case study illustrates a novel endoscopic surgical technique employing a large laterally-based flap as a posterior glottic keel by rotating it anteroinferiorly and suturing it in place. As a result of the procedure, the bilateral vocal folds, which were midline and immobile preoperatively, regained normal motion. The supraglottic airway was also restored. Successful endoscopic treatment of CS with bilateral vocal fold immobility is possible using a large laterally-based flap, even in the face of tissue changes secondary to chemoradiation.
\end{abstract}

\section{Keywords}

Posterior Glottic Stenosis, Supraglottic Stenosis, Combined Glottic Stenosis, Radiation, Endoscopic Surgery

\section{Introduction}

Posterior glottic stenosis (PGS) is a challenging diagnosis for physicians to manage. It usually occurs after laryngeal stenting and prolonged intubation. Isolated supraglottic stenosis (SS) is also difficult for physicians, traditionally occurring after surgery, caustic ingestion, trauma, severe infection, inflammatory disease and more 
recently secondary to chemoradiation. Historically, management of these stenoses was transcervical via a laryngofissure [1]-[3]. This approach resulted in significant patient morbidity, with aspiration, poor wound healing and laryngeal dysfunction. In response, more conservative endoscopic techniques were developed and are now most commonly performed [4]-[7].

Combined PGS and SS (CS) is occurring more frequently now as a late complication of chemotherapy and radiation treatment. Scarring and contracture affect both the supraglottis and posterior glottis. These cases are unique due to the severity of the stenosis, involvement of both the glottis and supraglottis and detrimental changes in the soft tissue secondary to chemoradiation. As was historically the case with PGS and SS, the transcervical approach via laryngofissure is used for treatment of CS. This is generally employed for stenosis secondary to trauma [8] [9]. However, this open approach is discouraged in patients who have had chemotherapy and radiation due to the morbidity of a laryngofissure, stenting and tracheotomy as well as the high risk of poor healing. As a result, a permanent tracheotomy is often recommended for these patients. As with PGS and SS, an endoscopic method for treatment of CS would be desirable to minimize patient morbidity. We present a case of severe CS with bilateral vocal fold immobility secondary to chemoradiation, successfully treated endoscopically by a novel technique employing a large laterally-based rotation flap.

\section{Case Report}

We present a 70-year-old female referred to our institution with a complaint of six months of dyspnea on exertion. She was no longer able to perform her activities of daily living secondary to the severity of symptoms. She had a history of oropharyngeal squamous cell carcinoma, treated 11 years prior by excision with post-operative chemotherapy and radiation. Examination revealed a patient with audible stridor in no acute distress. Her voice was mildly rough and slightly muted. Flexible laryngoscopy revealed a severe CS. Both vocal folds were immobile and the patient had a $3-4 \mathrm{~mm}$ airway. The findings were presumed secondary to progressive stenosis and fibrosis from her chemotherapy and radiation.

Although surgical treatment of the stenosis was necessary, considering the presence of significant damage to the laryngeal mucosa and surrounding tissues from radiation and chemotherapy, it was felt the risk of performing a laryngofissure with poor healing was too high, especially in the face of a relatively normal voice. Recognizing the success of treating such stenoses with larger local laryngopharyngeal flaps in transcervical surgery, it was decided to treat the stenosis with a larger flap as would be performed in open surgery, but via an endoscopic approach [1].

With this strategy in mind, the larynx was exposed using a Jako laryngoscope and the patient was ventilated via jet ventilation. A large laterally-based flap encompassing the tissue of the supraglottic stenosis as well as the mucosal and submucosal tissue overlying both arytenoids and the interarytenoid area was raised using a $\mathrm{CO}_{2}$ laser with an Accublade scanning attachment (Lumenis, Israel). Fibrotic tissue below the flap was excised and ablated. Care was taken not to injure the cricoarytenoid joint. After removal of the fibrosis the arytenoids appeared mobile to palpation. The mucosal flap was then rotated inferiorly and the distal end was sutured to the mucosal edge at the base of the left arytenoid using 5-0 vicryl sutures (Ethicon, Somerville, NJ) (Figure 1). With this positioning, the flap not only minimizes supraglottic contracture but also acts as a posterior keel preventing recurrent adhesions between the left and right arytenoid. A tracheotomy was not performed.

The peri-operative course was without complication. The patient is now 12 months post-operation. The patient's voice is improved, full vocal fold mobility has been restored bilaterally and the airway is stable with a normal caliber (Figure 2). The patient has returned to her usual activity prior to the development of symptoms.

\section{Discussion}

Traditionally, PGS was treated via laryngofissure with grafts or flaps for coverage of the excised scar tissue [2] [3]. These approaches were fraught with complications of poor wound healing due to the laryngofissure or graft failure. Since the advent of $\mathrm{CO}_{2}$ laser laryngeal surgery, PGS is most commonly treated endoscopically to avoid the morbidity of the transcervical approach [4] [6] [10]. These surgeries utilize small trapdoors or interarytenoid flaps to access the scar for its removal. The flap is then replaced in its original position for mucosal coverage to prevent restenosis. Isolated SS was also traditionally treated transcervically. As with PGS, more recent treatment of SS has been performed endoscopically. Transoral supraglottic laryngectomy or $\mathrm{CO}_{2}$ laser incision with radial balloon dilation have both been used with success [5] [7]. 


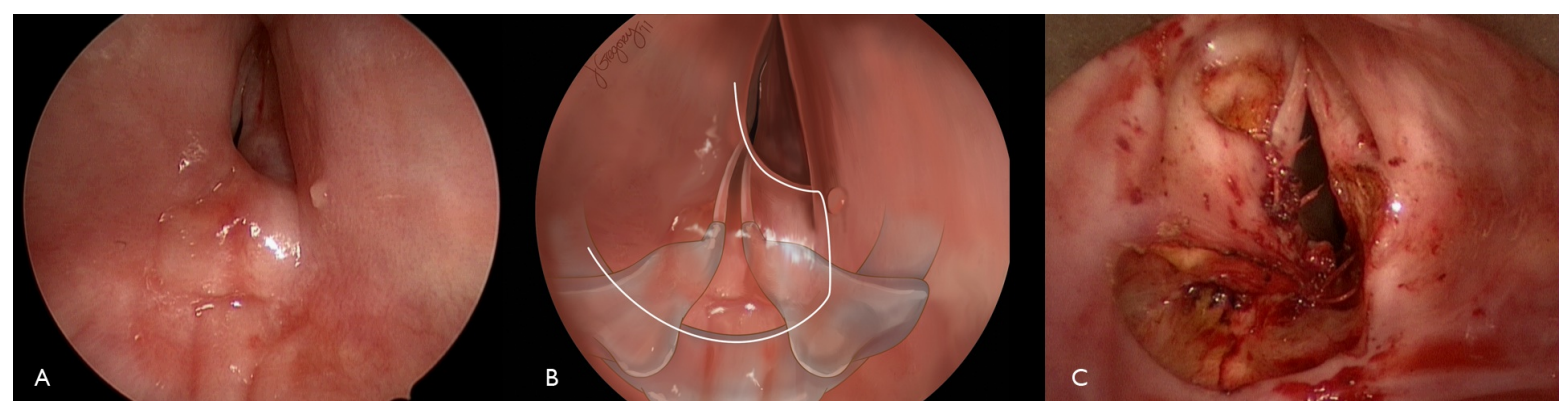

Figure 1. Intraoperative examination: (A) Preoperative (B) Artist rendition of flap outline and underlying anatomy, (C) Postoperative with flap inset.

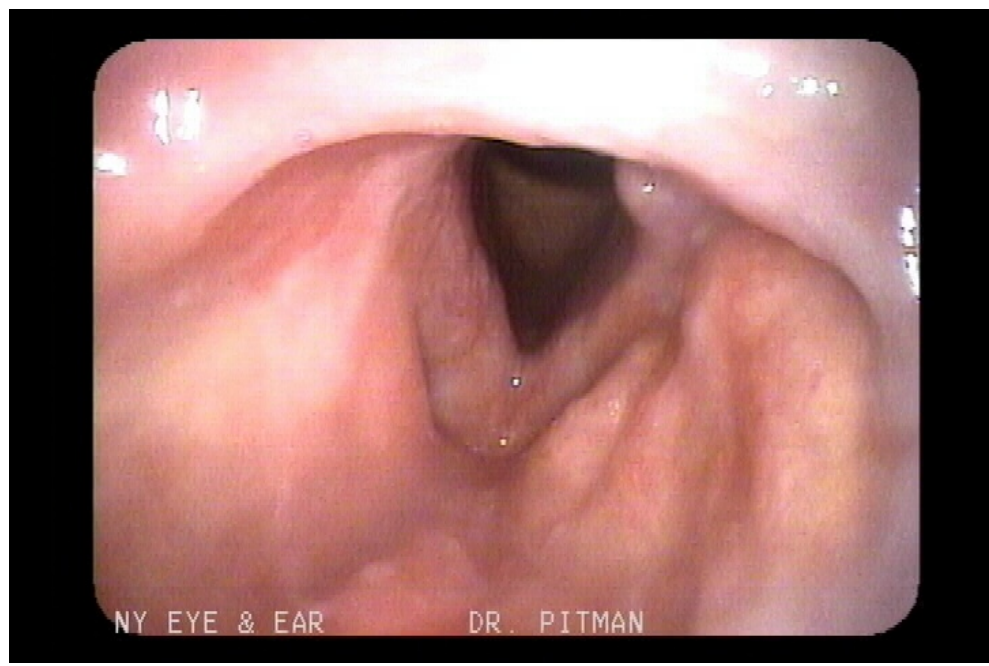

Figure 2. 12 months postoperative laryngoscopy, maximum abduction.

Unfortunately, many patients with chemoradiation-induced stenosis experience CS of the posterior glottis and supraglottis. In these patients, a transcervical approach via laryngofissure can be complicated by poor wound healing. The surgery also necessitates a stent, tracheotomy and second procedure for stent removal. This treatment approach results in significant patient morbidity [8] [9]. Due to the morbidity and high risk of poor wound healing in such transcervical procedures in the face of chemoradiation, many of these patients are treated with a permanent tracheotomy to bypass the stenosis.

The endoscopic surgical technique described herein can be successfully performed without a tracheotomy in patients with CS and bilateral vocal fold immobility secondary to chemoradation. This rotation flap differs from previous descriptions of endoscopic flaps described for isolated PGS [4] [6] [10]. The flap is larger and incorporates the supraglottis. It is also rotated inferiorly and sutured to the base of the arytenoid instead of simply being placed back into its preoperative position. This flap position results in coverage of one cricoarytenoid, joint minimizing the risk of local scar contracture and joint fibrosis. The flap also acts as a keel to decrease the risk of recurrence of CS. To our knowledge, such an endoscopic rotation flap has not been utilized previously for CS. This technique can be employed by otolaryngologists as an alternative to permanent tracheotomy or transcervical surgery with its increased morbidity and risk.

\section{Conclusion}

Treatment of CS via laryngofissure is a morbid procedure with a high risk of poor wound healing and further impairment of glottal function in patients who have been treated with chemotherapy and radiation. Successful endoscopic treatment of CS with bilateral vocal fold immobility is possible using a large laterally-based rotation flap, even in the face of tissue changes secondary to chemoradiation. This endoscopic procedure avoids the morbidity of a laryngofissure and decreases the risk of complications secondary to poor wound healing. 


\section{References}

[1] Montgomery, W.W. (1968) The Surgical Management of Supraglottic and Subglottic Stenosis. Annals of Otology, Rhinology, and Laryngology, 77, 534-546.

[2] Montgomery, W.W. (1973) Posterior and Complete Laryngeal (Glottic) Stenosis. Archives of Otolaryngology, 98, 170175. http://dx.doi.org/10.1001/archotol.1973.00780020178007

[3] Hoasjoe, D.K., Franklin, S.W., Aarstad, R.F., Day, T.A. and Stucker, F.J. (1997) Posterior Glottic Stenosis Mechanism and Surgical Management. Laryngoscope, 107, 675-679. http://dx.doi.org/10.1097/00005537-199705000-00022

[4] Dedo, H.H. and Sooy, C.D. (1984) Endoscopic Laser Repair of Posterior Glottic, Subglottic and Tracheal Stenosis by Division or Micro-Trapdoor Flap. Laryngoscope, 94, 445-450. http://dx.doi.org/10.1288/00005537-198404000-00001

[5] Krishna, P.D. and Malone, J.P. (2006) Isolated Adult Supraglottic Stenosis: Surgical Treatment and Possible Etiologies. American Journal of Otolaryngology, 27, 355-357. http://dx.doi.org/10.1016/j.amjoto.2005.11.021

[6] Simpson, C.B. and Rosen, C.A. (2008) Operative Techniques in Laryngology. Springer, Berlin.

[7] Vira, D., DeConde, A. and Chhetri, D.K. (2012) Endoscopic Management of Supraglottic Laryngopharyngeal Stenosis. Otolaryngology—Head and Neck Surgery, 146, 611-613. http://dx.doi.org/10.1177/0194599811431684

[8] Delaere, P.R., Ostyn, F., Segers, A. and Vandyck, W. (1991) Epiglottoplasty for Reconstruction of Posttraumatic Laryngeal Stenosis. Annals of Otology, Rhinology, and Laryngology, 100, 447-450.

[9] Doyle, P.J. (1980) Repair of Supraglottic Laryngeal Stenosis with Postcricoid Involvement. A Case Report. Annals of Otology, Rhinology, and Laryngology, 89, 576-577.

[10] Goldberg, A.N. (2000) Endoscopic Postcricoid Advancement Flap for Posterior Glottic Stenosis. Laryngoscope, 110, 482-485. http://dx.doi.org/10.1097/00005537-200003000-00029 